\title{
Situación actual de la intolerancia a la lactosa en la infancia
}

\author{
E. La Orden Izquierdo, I. Carabaño Aguado, FJ. Pelayo García \\ Unidad de Gastroenterología y Nutrición Infantil. Hospital Infanta Elena. Valdemoro, Madrid. España.
}

\begin{abstract}
Resumen
La lactosa es un disacárido de amplia distribución en la dieta y los productos farmacéuticos; es el azúcar de la leche de los mamíferos. Es frecuente una pérdida de la actividad lactásica intestinal con la edad, variable entre etnias y condicionada genéticamente, que puede causar su malabsorción. También puede ser secundaria a un daño mucoso intestinal y, en raras ocasiones, por déficit congénito. Malabsorción no implica necesariamente una intolerancia con significación clínica. La exclusión de la lactosa de la dieta produce mejoría clínica pero, a largo plazo, puede conllevar un defecto en la ingesta diaria recomendada de otros nutrientes como el calcio. Puede ser reintroducida en la dieta, si bien el umbral clínico de tolerancia es individual.
\end{abstract}

Palabras clave: Intolerancia a lactosa. Malabsorción.

\section{Current status of lactose intolerance in children}

\begin{abstract}
Lactose is a widely distributed disaccharide in the diet and pharmaceutical industries. It is the sugar from the milk of mammals. The loss of intestinal lactase activity with age is frequent. It varies between ethnic and genetic conditions, and may cause its malabsorption. It may also be secondary to intestinal mucosal damage and, rarely, to congenital deficiency. Malabsorption does not necessarily imply clinical intolerance, with gastrointestinal symptoms after its ingestion. The exclusion of lactose from the diet produces clinical improvement but it could lead to a defect in the recommended daily intake of other nutrients such as calcium. It can be reintroduced into the diet but there is an individual clinical threshold of tolerance.
\end{abstract}

Key words: Lactose intolerance. Malabsorption.

\section{Introducción}

La intolerancia digestiva a la lactosa es una entidad de diagnóstico frecuente en la edad pediátrica. Este término engloba de manera general distintas situaciones fisiopatológicas. La aparición de sintomatología digestiva tras su ingesta constituye la sospecha principal para su diag-

Enrique la Orden Izquierdo, e_o_izquierdo@yahoo.es

Los autores declaran no presentar conflictos de intereses en relación con la preparación y publicación de este artículo. 
nóstico y la mejoría clínica tras la exclusión, parcial o total, de la lactosa de la dieta suele ser el método de confirmación más usado en la práctica diaria. Sin embargo, su incidencia real puede estar sobreestimada en este grupo etario, y su restricción dietética precoz y prolongada puede tener consecuencias a largo plazo tales como osteoporosis, entre otros.

\section{Aspectos dietéticos de la lactosa}

La lactosa es un disacárido resultante de la unión de glucosa y galactosa a través de un enlace $\beta(1 \rightarrow 4)$. Es el azúcar predominante de la leche de los mamíferos y de sus derivados'. Se obtiene a partir de suero lácteo tras procesos de ultrafiltración, evaporación y cristalización en forma anhidra o hidratada. Se emplea en la elaboración de productos dietéticos, como sustituto a la sacarosa en productos horneados, y como excipiente de algunos fármacos. Entre sus funciones, además de aportar energía, facilita la absorción de calcio y el desarrollo de una microbiota intestinal grampositiva (bifidobacterias) tras su fermentación, que impide el crecimiento de gérmenes patógenos ${ }^{2}$.

\section{Absorción de la lactosa. Aspectos genéticos}

La lactosa llega indemne al intestino delgado proximal, donde es degradada enzimáticamente por la lactasa en sus componentes. Ambos monosacáridos se absorben a continuación a través de transporte activo acompañado de dos moléculas de $\mathrm{Na}^{+}$(mediado por el transportador SGLUT1 hacia el interior del enteriocito) y difunden a la sangre de forma pasiva o mediante el transportador GLUT2 ${ }^{3}$. La galactosa debe transformarse en glucosa mediante la galactoquinasa (su déficit origina la aparición de cataratas bilaterales) y la galactosa1-Pi-uridiltransferasa (su déficit origina la galactosemia clásica) para poder ser utilizada $^{4}$. La lactosa no absorbida es una sustancia osmóticamente activa que atrae líquido y electrolitos hacia la luz intestinal. Acaba siendo hidrolizada y fermentada por la flora colónica, produciendo gas (hidrógeno y metano) que se elimina por la respiración, y ácidos orgánicos de cadena corta, como el butirato (principal combustible del colonocito), el ácido propiónico, el acético y el láctico, que acidifican el pH colónico'.

La lactasa es una $\beta$-galactosidasa que se expresa preferentemente en el yeyuno y disminuye de manera progresiva hacia el íleon, estableciéndose este gradiente desde la semana ocho de gestación. Su actividad aumenta progresivamente, sobre todo en el tercer trimestre de gestación, siendo má- 
xima al nacer ${ }^{5,6}$. Dietas con elevado contenido en almidón aumentan los niveles de ARN mensajero (ARNm) y la cantidad de lactasa, mientras que dietas con contenido elevado en triglicéridos de cadena larga disminuyen la expresión del gen?. Su pH óptimo de actuación es 6-8. Otros sustratos que hidroliza son la lactosiceramina, la celobiosa y la cetroliosa ${ }^{1}$. La actividad lactásica puede descender a partir de los 3-5 años de vida hasta un 5-10\% de su actividad en la edad adulta. Esto ocurre por disminución de su síntesis mediada por menor expresión génica, más que por un bajo consumo de lácteos como se creía anteriormente (inducción por sustrato). La hipolactasia del adulto presenta variaciones étnicas y geográficas, y es máxima en la raza asiática $(90 \%)$ y en afroamericanos $(80 \%)$, y menor en hispanoamericanos (53\%) y blancos no hispanos (15$25 \%)^{8}$. Se han planteado hipótesis que intentan explicar esta diversidad geográfica atendiendo, por ejemplo, al papel de la lactosa en la absorción de cal- cio en los países situados a altas latitudes o en la protección frente a la malaria por Plasmodium falciparum ${ }^{6,9}$.

El gen de la lactasa-floricina-hidrolasa se localiza en el cromosoma 2q21. En 2002, Enattah et al. identificaron el polimorfismo C/T-13910 del gen de la lactasa basado en la presencia de una citosina o una timidina en posición 13910. La variante en homocigosis C/C sería la asociada a la no persistencia de lactasa (fenotipo intolerante), mientras que la variante $C / T$ o $T / T$ estaría ligada a la persistencia de la actividad lactásica ${ }^{6}$. Otro polimorfismo descrito sería el G/A-22018: la homocigosis G/G (guanina/guanina) se asociaría a la no persistencia, mientras que las variantes G/A y A/A (adenina/adenina) las presentarían los fenotipos lactasa-persistentes (tabla 1). Los mecanismos responsables incluirían un descenso en la producción del ARNm, una alteración en la transcripción génica o en la traducción, o incluso una disminución del número de enterocitos fabricantes de lactasa'.

Tabla 1. Genética de la lactasa-floricina-hidrolasa

\begin{tabular}{l|l|l}
\hline & Persistencia de lactasa & No persistencia de lactasa \\
\hline Posición 13910 & C/T y T/T & C/C \\
\hline Posición 22018 & G/A y A/A & G/G \\
\hline
\end{tabular}

C: citosina; T: timina; G: guanina; A: adenina. 
Definiciones $^{10}$

- Intolerancia clínica a la lactosa: síndrome clínico que cursa con dolor abdominal, diarrea y meteorismo tras la ingesta de una cantidad variable de lactosa (el umbral de tolerancia varía de modo individual).

- Malabsorción de lactosa: situación fisiopatológica caracterizada por una pobre hidrólisis de la lactosa por una actividad lactásica deficiente o una sobrecarga oral excesiva de este disacárido. Puede cursar con o sin intolerancia clínica.

- Hipolactasia primaria: también llamada hipolactasia del adulto, hipolactasia hereditaria, no persistencia de lactasa. Consiste en una pérdida de la actividad lactásica intestinal con la edad, variable según etnia y condicionada genéticamente que obedece a múltiples mecanismos.

- Hipolactasia secundaria: pérdida de la actividad lactásica intestinal secundaria a un daño mucoso por otro motivo: gastroenteritis aguda y síndrome postenteritis, enfermedad inflamatoria intestinal, malnutrición proteico-calórica que curse con atrofia vellositaria, etc. Re- suelta la causa primaria, y descartada una hipolactasia primaria, la actividad enzimática suele recuperarse en el enterocito.

- Hipolactasia del desarrollo: es la deficiencia relativa de actividad lactásica con o sin defecto en otras disacaridasas por inmadurez intestinal en los neonatos menores de 34 semanas de gestación. Tiene carácter transitorio. La lactosa no hidrolizada es fermentada en el colon, lo cual acidifica el pH fecal y permite el crecimiento de una flora colónica favorable en el intestino en desarrollo.

- Hipolactasia/alactasia congénita: entidad rara debida a un déficit congénito de actividad lactásica con o sin una mínima actividad residual. Cursa como una diarrea grave e importantes alteraciones hidroelectrolíticas tras la introducción de leche materna o humanizada convencional. Su diagnóstico se basa en la realización de una biopsia intestinal (estudio histopatológico normal) y cuantificación de actividad de disacaridasas de pared (actividad lactásica mínima o nula). Sin tratamiento (dieta exenta de lactosa) su pronóstico es fatal. 


\section{Clínica}

Los síntomas típicos de intolerancia a la lactosa incluyen la presencia de dolor abdominal, flatulencia, diarrea, meteorismo, náuseas y vómitos por el efecto osmótico intestinal y posterior fermentación colónica de la lactosa. En algunas ocasiones puede aparecer estreñimiento por descenso de la motilidad gastrointestinal en probable relación con la presencia de flora productora de metano. El umbral de tolerancia clínica individual dependerá, además, de la actividad lactásica, del tiempo de tránsito intestinal y de la capacidad de la flora colónica del individuo para fermentar la lactosa. Es importante realizar un adecuado diagnóstico diferencial con otras patologías que cursen de manera similar, como sobrecrecimiento bacteriano, alergia a proteínas de leche de vaca no mediada por IgE, etc. En pacientes en los que la clínica no remita tras exclusión de la lactosa de la dieta, hay que sospechar otra causa subyacente, como un síndrome del intestino irritable ${ }^{6}$.

\section{Diagnóstico}

Existen distintos métodos para valorar la persistencia de actividad lactásica intestinal:

- Control de glucemia a los $30 \mathrm{mi}$ nutos de una sobrecarga oral de lactosa (elevada si se produce absorción de la misma) ${ }^{6}$ : indica hidrólisis de lactosa y su absorción posterior, no siendo sensible para el estudio definitivo de malabsorción ${ }^{10}$.

- Respuesta a la exclusión de lactosa de la dieta durante dos semanas (mejoría clínica) y prueba de provocación posterior ${ }^{10}$. Permite diagnosticar a los pacientes intolerantes pero no al paciente que curse con malabsorción asintomática.

- Test de hidrógeno espirado: la lactosa no absorbida es fermentada por la flora colónica produciendo hidrógeno y metano, que se eliminan en el aire exhalado. Hasta en un $20 \%$ de la población la flora intestinal no genera hidrógeno, por lo que tienen especial interés las pruebas que detectan tanto hidrógeno como metano. Si tras la toma de $50 \mathrm{~g}$ de lactosa se produce una elevación de la línea de base del hidrógeno espirado por encima de 20 partes por millón (ppm) es indicativo de malabsorción de lactosa (una elevación precoz a la hora haría pensar en la posibilidad de un sobrecrecimiento bacteriano). La malabsorción puede cursar o no con clínica digestiva ${ }^{11}$. El uso de 
"cantidades habituales" de lactosa (por ejemplo, la contenida en un vaso de leche o en un lácteo fermentado) podría ser de mayor utilidad para su realización en vez del uso de una solución acuosa estándar de lactosa para marcar el umbral de tolerancia clínica individual'.

- Estudio genotípico: no disponible en la práctica habitual. Su principal utilidad sería diferenciar aquellos casos de intolerancia a la lactosa de las hipolactasias secundarias ${ }^{6}$.

- El estudio de la actividad enzimática de las disacaridasas intestinales (entre ellas la lactasa) en muestra de biopsia yeyunal permite el diagnóstico directo, pero su sensibilidad es menor a la proporcionada por el test de hidrógeno espira$\mathrm{do}^{6}$.

\section{Manejo}

Su tratamiento se basa en la exclusión de lactosa durante al menos cuatro semanas. Posteriormente, puede ser reintroducida en pequeñas cantidades para permitir la adaptación de la flora colónica. Los cambios en la dieta que afecten al vaciamiento gástrico (temperatura, osmolaridad de la mezcla gástrica, presencia de fibra o cereales...) puede au- mentar el tiempo de permanencia de la lactosa en el intestino delgado y mejorar su hidrólisis ${ }^{6}$. La lactosa está ampliamente difundida en la industria alimentaria y farmacéutica y su exclusión estricta puede ser complicada. En la leche y derivados lácteos el contenido de lactosa varía en función del grado de fermentación y procesamiento. También su contenido en calcio es variable. Existen fórmulas sin lactosa que están indicadas en estas situaciones ${ }^{10}$. Es importante este aspecto de cara a su posterior reintroducción en la dieta.

La exclusión dietética de la lactosa puede exacerbar el riesgo de osteoporosis en personas con factores de riesgo (no en personas sanas): mujeres, grupos étnicos seleccionados, patologías que cursen con un defecto en la absorción intestinal de calcio y vitamina $D$ (enfermedad inflamatoria intestinal) o un aumento en la resorción ósea (tratamiento con corticoides). El efecto no sería tanto por un déficit en la absorción de calcio sino por un defecto en su ingesta y en la de otros micronutrientes como la vitamina $D^{12,13}$. También puede tener efectos sobre el riesgo cardiovascular y desarrollo de pólipos colónicos adenomatosos en relación con un descenso en la producción de butirato ${ }^{12}$.

La prevalencia de sobrecrecimiento bacteriano o aumento en la flora fer- 
mentadora intestinal entre los pacientes con síntomas sugerentes de intolerancia a la lactosa es elevada, por lo que este diagnóstico diferencial debe ser considerado para evitar la instauración de una dieta empírica restrictiva ${ }^{14}$.

La evidencia disponible sugiere que los adultos y adolescentes diagnosticados de malabsorción de lactosa podrían ingerir al menos $12 \mathrm{~g}$ de lactosa de una vez; sin embargo, no se ha descrito la dosis mínima en niños ${ }^{12}$. El manejo incluiría el consumo regular de lácteos para mejorar la adaptación de la flora colónica facilitando la digestión de la lactosa, el consumo de lácteos con menor contenido en lactosa (fermentados o tratados enzimáticamente con $\beta$-galactosidasa) o adicionados con enzimas bacterianas capaces de digerir la lactosa ${ }^{13,15}$. El uso de fermentos lácticos (Lactobacillus casei y S. thermophilus) presentes en el yogur de manera continuada durante seis meses puede mejorar la tolerancia a la lactosa, si bien el modo de actuación no está bien clarificado.

\section{Conclusiones}

La intolerancia clínica a la lactosa puede estar sobrediagnosticada en la infancia. El déficit congénito es muy infrecuente, su déficit secundario suele ser transitorio y el déficit primario asociado a la edad no necesariamente es sintomático. El tratamiento consiste en una dieta baja en lactosa en los casos leves y exenta de lactosa en los casos graves. La lactosa está ampliamente difundida, tanto en productos dietéticos como farmacológicos. La posterior reintroducción progresiva y controlada de lactosa en la dieta puede ayudar a conocer el umbral clínico individual de tolerancia. El uso de fermentos lácticos y modificaciones dietéticas concomitantes pueden ayudar a mejorar este umbral. Es importante recordar suplementar el calcio en dietas exentas de lácteos durante la infancia o en presencia de factores de riesgo de osteoporosis para asegurar una adecuada mineralización ósea.

\section{Bibliografía}

1. Infante $D$. Intolerancia a la lactosa: en quién y por qué. An Pediatr (Barc). 2008;69(2):103-5.

2. Guerra Hernández EJ. Azúcares, miel y productos de confitería. En: Sociedad Española de Nutrición Parenteral y enteral, ed. Tratado de Nutri- ción. Tomo II. Composición y calidad nutritiva de los alimentos. 2. ${ }^{\text {a }}$ ed. Madrid: Editorial Médica Panamericana; 2010. p. 221-48.

3. Martínez de Victoria Muñoz E, Mañas Almendros M, Yago Torregrosa MD. Fisiología de la digestión. En: Sociedad Española de Nutrición Parenteral y enteral, ed. Tratado de Nutrición. Tomo 
I. Bases Fisiopatológicas y bioquímicas de la nutrición. 2. ed. Madrid: Editorial Médica Panamericana; 2010. p. 171-201.

4. Ruiz Pons M, Sánchez-Valverde Visus F, Dalmau Serra J, Gómez López L. Errores innatos del metabolismo de los carbohidratos. En: Tratamiento nutricional de los errores innatos del metabolismo. 2. ${ }^{a}$ ed. Madrid: Drug Farma S.L.; 2007. p. 39-85.

5. Raul F, Lacroix B, Aprahamian M. Longitudinal distribution of brush border hydrolases and morphological maturation in the intestine of the preterm infant. Early Hum Dev. 1986;13(2):225-34.

6. Lomer MCE, Parkes GC, Sanderson D. Review article: lactose intolerance in clinical practice-myths and realities. Aliment Pharmacol Ther. 2008;27:93-103.

7. Gil Hernández A, Aguilera García C, Gómez Llorente $C$. Nutrigenómica. En: Sociedad Española de Nutrición Parenteral y enteral, ed. Tratado de Nutrición. Tomo I. Bases Fisiopatológicas y bioquímicas de la nutrición. 2. ${ }^{a}$ ed. Madrid: Editorial Médica Panamericana; 2010. p. 749-806.

8. Sahi T. Genetics and epidemiology of adulttype hypolactasia. Scand J Gastroenterol. 1994; 202(Suppl):7-20.

9. Lokki Al, Järvela I, Israelsson E, Maiga B, Troye-Blomberg $M$, Dolo $A$ et al. Lactasa persistence genotypes and malaria susceptibility in Fulani of Mali. Malar J. 2011;10(1):9.

10. Heyman MB. Lactose intolerance in infants, children, and adolescents. Pediatrics. 2006;118(3): 1279-86.

11. Braden B. Methods and functions: Breath test. Best Pract Res Clin Gastroenterol. 2009;23: 337-52.

12. Suchy FJ, Brannon PM, Carpenter TO, Fernandez JR, Gilsanz V, Gould JB et al. NIH Consensus Development Conference Statement: Lactose Intolerance and Health. NIH Consens State Sci Statements. 2010;27(2):1-27.

13. Savaiano D. Lactose intolerance: an unnecessary risk for low bone density. Nestle Nutr Workshop Ser Pediatr Program. 2011;67:161 71.

14. Novillo $A$, Peralta $D$, Dima $G$, Besasso $H$, Soifer $\mathrm{L}$. Frequency of bacterial overgrowth in patients with clinical lactose intolerance. Acta Gastroenterol Latinoam. 2010;40(3):221-4.

15. Baró Rodríguez L, Lara Villoslada F, Corral Román E. Leche y derivados lácteos. En: Sociedad Española de Nutrición Parenteral y enteral, ed. Tratado de Nutrición. Tomo II. Composición y calidad nutritiva de los alimentos. 2. ${ }^{\mathrm{a}}$ ed. Madrid: Editorial Médica Panamericana; 2010. p. 1-26. 